OPEN ACCESS

Edited by:

Weimin Ye

Karolinska Institutet (KI), Sweden

Reviewed by:

Diego A. Moreno,

CEBAS-CSIC, Food Science and Technology Department (Spanish

National Research Council), Spain

Wenbin Liang,

Fujian Medical University, China

*Correspondence:

$X$ in $X u$

drxuxin@zju.edu.cn

Specialty section: This article was submitted to Nutritional Epidemiology, a section of the journal

Frontiers in Nutrition

Received: 24 March 2021 Accepted: 14 June 2021

Published: 05 July 2021

Citation:

Xu X, Li S and Zhu Y (2021) Dietary Intake of Tomato and Lycopene and Risk of All-Cause and Cause-Specific Mortality: Results From a Prospective

Study. Front. Nutr. 8:684859.

doi: 10.3389/fnut.2021.684859

\section{Dietary Intake of Tomato and Lycopene and Risk of All-Cause and Cause-Specific Mortality: Results From a Prospective Study}

\author{
Xin Xu*, Shiqi Li and Yi Zhu \\ Department of Urology, The First Affiliated Hospital, School of Medicine, Zhejiang University, Hangzhou, China
}

Evidence on the relationship between consumption of tomato or lycopene and mortality is limited. We investigated the associations of raw tomato, tomato catsup or lycopene intake with all-cause and cause-specific mortality using data from the Prostate, Lung, Colorectal, and Ovarian (PLCO) screening trial. A multivariate Cox proportional hazards model was used to estimate hazard ratios (HRs) and 95\% confidence intervals (Cls). During a total of 1,672,715 follow-up years, 24,141 all-cause deaths, 7,534 cardiovascular disease (CVD) deaths and 7,161 cancer deaths occurred. Total mortality was statistically significantly inversely associated with intake of raw tomato (Q5 vs. Q1; $\mathrm{HR}, 0.95$ [95\% Cl, 0.91-0.99]), tomato catsup (Q5 vs. Q1; HR, 0.93 [95\% Cl, 0.89-0.97]), and moderate lycopene (Q4 vs. Q1; HR, 0.88 [95\% Cl, 0.85-0.93]). CVD mortality was significantly inversely related with intake of moderate raw tomato (Q4 vs. Q1; HR, 0.90 [95\% Cl, 0.83-0.97]), tomato catsup (Q5 vs. Q1; HR, 0.92 [95\% Cl, 0.85-0.99]), and moderate lycopene (Q4 vs. Q1; HR, 0.90 [95\% Cl, 0.83-0.98]). Dietary intake of raw tomato (Q5 vs. Q1; HR, 1.04 [95\% Cl, 0.96-1.14]) and tomato catsup (Q5 vs. Q1; $\mathrm{HR}, 1.00$ [95\% Cl, 0.93-1.08]) were not related with cancer mortality. Moderate dietary intake of lycopene was significantly associated with a lower cancer mortality (Q4 vs. Q1; $\mathrm{HR}, 0.89$ [95\% Cl, 0.82-0.96]). There was a non-linear J-shaped association between consumption of raw tomato, tomato catsup or lycopene and total mortality ( $P$ for nonlinearity $<0.001$ ). In conclusion, in this large nationally representative sample of US adult population, tomato products, and lycopene intake were associated with lower risks of total and CVD mortality. Moderate consumption of lycopene was also related with a reduced cancer mortality. Further clinical studies and dietary intervention studies are warranted to confirm our premilitary findings.

Keywords: tomato, lycopene, mortality, cohort, PLCO

\section{INTRODUCTION}

Tomato and tomato products have been widely recognized as healthy foods because of their high content of lycopene. Human trials have shown the potential of dietary lycopene or lycopene supplement in reducing levels of proinflammatory mediators and oxidative stress (1), regulating cardiovascular variables (2) and blood pressure (3), and improving the lipid profile (4). 
TABLE 1 | Main characteristic of participants included in this study by raw tomato intake.

\begin{tabular}{|c|c|c|c|c|c|c|}
\hline Variables & Q1 $(n=20,508)$ & $\mathrm{Q} 2(n=20,276)$ & Q3 $(n=20,619)$ & Q4 $(n=20,928)$ & Q5 $(n=19,506)$ & $p$-value \\
\hline Age $(y)$, mean $(S D)$ & $62.2(5.4)$ & $62.4(5.3)$ & $62.5(5.3)$ & $62.6(5.2)$ & $62.4(5.2)$ & $<0.001$ \\
\hline \multicolumn{7}{|l|}{$\operatorname{Sex}(n, \%)$} \\
\hline Male & $11,349(55.3 \%)$ & 10,239 (50.5\%) & $9,433(45.7 \%)$ & $8,694(41.5 \%)$ & $9,818(50.3 \%)$ & $<0.001$ \\
\hline Female & $9,158(44.7 \%)$ & 10,037 (49.5\%) & 11,186 (54.3\%) & $12,232(58.5 \%)$ & $9,686(49.7 \%)$ & \\
\hline \multicolumn{7}{|l|}{ Smoking status ( $n, \%)$} \\
\hline Never & $9,380(45.7 \%)$ & 9,704 (47.9\%) & 10,146 (49.2\%) & $10,289(49.2 \%)$ & $9,077(46.5 \%)$ & $<0.001$ \\
\hline Current & $2,358(11.5 \%)$ & 1,994 (9.8\%) & 1,694 (8.2\%) & $1,710(8.2 \%)$ & $1,656(8.5 \%)$ & \\
\hline Former & 8,766 (42.8\%) & 8,575 (42.3\%) & $8,770(42.6 \%)$ & $8,926(42.7 \%)$ & 8,767 (45.0\%) & \\
\hline \multicolumn{7}{|l|}{ Education $(n, \%)$} \\
\hline$\leq$ High school & $9,332(45.5 \%)$ & 8,767 (43.2\%) & $8,299(40.2 \%)$ & 8,657 (41.4\%) & $7,912(40.6 \%)$ & $<0.001$ \\
\hline$\geq$ Some college & $11,130(54.3 \%)$ & 11,460 (56.5\%) & 12,277 (59.5\%) & 12,234 (58.5\%) & 11,560 (59.3\%) & \\
\hline \multicolumn{7}{|l|}{ BMI $(n, \%)$} \\
\hline$<25.0 \mathrm{~kg} / \mathrm{m}^{2}$ & 6,925 (33.8\%) & 6,895 (34.0\%) & $7,154(34.7 \%)$ & $7,257(34.7 \%)$ & $6,248(32.0 \%)$ & $<0.001$ \\
\hline$\geq 25.0 \mathrm{~kg} / \mathrm{m}^{2}$ & $13,281(64.8 \%)$ & 13,104 (64.6\%) & $13,223(64.1 \%)$ & $13,406(64.1 \%)$ & $12,990(66.6 \%)$ & \\
\hline \multicolumn{7}{|l|}{ Race $(n, \%)$} \\
\hline White, non-Hispanic & $17,582(85.7 \%)$ & $18,341(90.5 \%)$ & 19,143 (92.8\%) & 19,475 (93.1\%) & 18,056 (92.6\%) & $<0.001$ \\
\hline Other & $2,915(14.2 \%)$ & $1,928(9.5 \%)$ & $1,472(7.1 \%)$ & $1,444(6.9 \%)$ & $1,439(7.4 \%)$ & \\
\hline \multicolumn{7}{|l|}{ Alcohol drinking status $(n, \%)$} \\
\hline Never & $1,991(9.7 \%)$ & $1,997(9.8 \%)$ & $2,011(9.8 \%)$ & $2,143(10.2 \%)$ & $1,982(10.2 \%)$ & $<0.001$ \\
\hline Former & $3,499(17.1 \%)$ & $3,010(14.8 \%)$ & $2,701(13.1 \%)$ & $2,875(13.7 \%)$ & $2,684(13.8 \%)$ & \\
\hline Current & 14,359 (70.0\%) & 14,703 (72.5\%) & $15,391(74.6 \%)$ & 15,305 (73.1\%) & 14,302 (73.3\%) & \\
\hline Total energy intake (kcal/d), mean (SD) & $1554.1(711.8)$ & $1635.6(690.4)$ & $1714.1(683.7)$ & $1784.6(707.5)$ & 2015.8 (803.6) & $<0.001$ \\
\hline
\end{tabular}

Y, year; SD, standard deviation; BMI, body mass index.

Epidemiological studies have shown inverse associations between consumption of tomato or lycopene and the risk of developing several major chronic diseases, including incidence of cardiovascular disease (CVD) $(5,6)$, metabolic syndrome $(7,8)$, dementias (9), and some types of cancer (10-13). There is an increasing interest in the association between tomato or lycopene intake and mortality. To the best of our knowledge, currently only one cohort study has been published on this topic, which reported an inverse relationship between tomato intake and both total and CVD mortality (14). However, this study failed to distinguish raw tomatoes and processed tomatoes, which may have differential effects on health outcomes. In addition, this study did not further explore the potential doseresponse relationship between tomato or lycopene consumption and mortality.

To provide evidence to bridge this knowledge gap, we investigated the associations of raw tomato, tomato catsup or lycopene intake with all-cause and cause-specific mortality using data from the Prostate, Lung, Colorectal, and Ovarian (PLCO) screening trial.

\section{METHODS}

\section{Subjects and Study Design}

The design and methods of the PLCO screening trial have been previously described (15). Briefly, the PLCO study is a randomized, controlled trial to assess whether certain screening tests reduce death from prostate, lung, colorectal, and ovarian cancer. PLCO consisted of $\sim 155,000$ participants aged 55-74 years and enrolled between November 1993 and July 2001. The participants were from 10 clinical screening centers throughout the United States. PLCO study was approved by the institutional review boards of the National Cancer Institute and each of the participating centers. Informed consent was obtained from each eligible participant in the study. The ClinicalTrials.gov numbers for PLCO are NCT00002540, NCT01696968, NCT01696981, and NCT01696994.

\section{Data Collection and Dietary Assessment}

All participants were asked to complete a baseline questionnaire (BQ) containing baseline information such as demographics and medical history. The Dietary History Questionnaire (DHQ) was administered to participants to collect dietary data. DHQ included the prespecified portion size and consumption frequency of 124 food items and supplement use over the previous year (16). The USDA 1994-1996 Continuing Survey of Food Intakes by Individuals (17) were used to calibrate DHQ data and calculate the daily intake of tomato products and lycopene.

\section{Participant Selection}

Participants were omitted from this study if they did not complete a BQ $(n=4,918)$; had reported a previous cancer at baseline $(n=$ $10,199)$; did not have follow-up time $(n=12)$; failed to complete 
TABLE 2 | Associations between intake of raw tomato, tomato catsup or lycopene, and total mortality.

\begin{tabular}{|c|c|c|c|c|c|}
\hline Variables & Median & Cohort (n) & Cases (n) & HR $(95 \% \mathrm{Cl})^{\#}, p$-value & HR $(95 \% \mathrm{Cl})^{*}, p$-value \\
\hline \multicolumn{6}{|l|}{ Raw tomato (g/day) } \\
\hline Q1 ( $\leq 3.63)$ & 1.63 & 20,508 & 5,288 & Reference group & Reference group \\
\hline Q2 ( $\geq 3.65-\leq 9.53)$ & 6.33 & 20,276 & 4,872 & $0.91(0.88-0.95), p<0.001$ & $0.96(0.92-0.99), p=0.027$ \\
\hline Q3 ( $\geq 9.55-\leq 17.56)$ & 12.91 & 20,619 & 4,669 & $0.86(0.83-0.90), p<0.001$ & 0.93 (0.89-0.96), $p<0.001$ \\
\hline Q4 ( $\geq 17.67-\leq 32.44)$ & 23.79 & 20,928 & 4,658 & 0.85 (0.82-0.89), $p<0.001$ & $0.91(0.87-0.95), p<0.001$ \\
\hline \multirow[t]{2}{*}{ Q5 ( $\geq 32.64)$} & 50.24 & 19,506 & 4,654 & $0.91(0.87-0.94), p<0.001$ & $0.95(0.91-0.99), p=0.026$ \\
\hline & & & & $p$ for trend $=0.001$ & $p$ for trend $=0.141$ \\
\hline \multicolumn{6}{|c|}{ Tomato catsup (g/day) } \\
\hline Q1 $(\leq 0.11)$ & 0 & 21,636 & 5,501 & Reference group & Reference group \\
\hline Q2 $(\geq 0.13-\leq 0.44)$ & 0.17 & 19,575 & 4,306 & $0.92(0.89-0.96), p<0.001$ & $0.94(0.90-0.98), p=0.004$ \\
\hline Q3 ( $\geq 0.48-\leq 1.15)$ & 0.58 & 21,243 & 4,540 & $0.88(0.85-0.92), p<0.001$ & $0.91(0.87-0.94), p<0.001$ \\
\hline Q4 ( $\geq 1.20-\leq 2.53)$ & 1.99 & 19,486 & 5,057 & 0.91 (0.88-0.95), $p<0.001$ & $0.93(0.90-0.97), p=0.001$ \\
\hline \multirow[t]{2}{*}{ Q5 ( $\geq 2.95)$} & 5.06 & 19,897 & 4,737 & $0.95(0.91-0.99), p=0.013$ & $0.93(0.89-0.97), p=0.001$ \\
\hline & & & & $p$ for trend $=0.923$ & $p$ for trend $=0.080$ \\
\hline \multicolumn{6}{|l|}{ Lycopene (mg/day) } \\
\hline Q1 (<2.79) & 2.07 & 20,368 & 5,417 & Reference group & Reference group \\
\hline Q2 ( $\geq 2.79-<4.06)$ & 3.42 & 20,367 & 4,722 & $0.89(0.86-0.93), p<0.001$ & $0.92(0.89-0.96), p<0.001$ \\
\hline Q3 ( $\geq 4.06-<5.61)$ & 4.76 & 20,368 & 4,509 & $0.87(0.84-0.91), p<0.001$ & 0.91 (0.87-0.95), $p<0.001$ \\
\hline Q4 ( $\geq 5.61-<8.44)$ & 6.74 & 20,367 & 4,430 & 0.87 (0.83-0.90), $p<0.001$ & 0.88 (0.85-0.93), $p<0.001$ \\
\hline \multirow[t]{2}{*}{ Q5 ( $\geq 8.44)$} & 12.06 & 20,367 & 5,063 & $1.00(0.96-1.04), p=0.947$ & $0.99(0.94-1.04), p=0.627$ \\
\hline & & & & $p$ for trend $=0.012$ & $p$ for trend $=0.203$ \\
\hline
\end{tabular}

\#Adjusted for age (continuous) and sex (male vs. female).

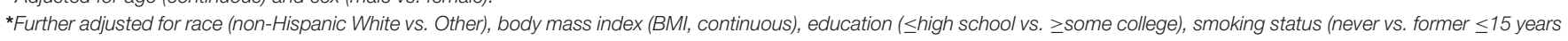

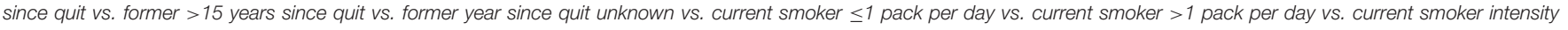

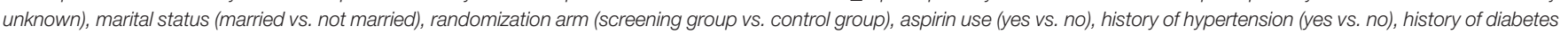

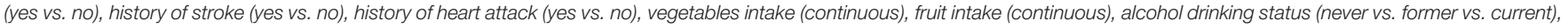
and total energy intake (continuous).

DHQ or the DHQ was not valid $(n=37,936)$. Finally, our study included a total of 101,832 individuals.

\section{Outcome Assessment}

Study participants were followed from the date of DHQ completion to the time of death or through 2015. Deaths were identified by the administration of the Annual Study Update (ASU) questionnaires, reports from relatives, friends, or physicians, and National Death Index (NDI) Plus searches. The cause of deaths was classified according to the International Classification of Diseases, 9th Revision (ICD-9). The primary outcomes of interest were all-cause mortality (death from any cause) and mortality from CVD or cancer.

\section{Statistical Analysis}

Tomato or lycopene consumption was categorized into five equal groups. A multivariate Cox proportional hazards model was used to estimate hazard ratios (HRs) and 95\% confidence intervals (CIs). Two models were established to adjust for variables. Model 1 was adjusted for age (continuous) and sex (male vs. female). Model 2 was further adjusted for race (nonHispanic White vs. Other), body mass index (BMI, continuous), education ( $\leq$ high school vs. $\geq$ some college), smoking status (never vs. former $\leq 15$ years since quit vs. former $>15$ years since quit vs. former year since quit unknown vs. current smoker $\leq 1$ pack per day vs. current smoker $>1$ pack per day vs. current smoker intensity unknown), marital status (married vs. not married), randomization arm (screening group vs. control group), aspirin use (yes vs. no), history of hypertension (yes vs. no), history of diabetes (yes vs. no), history of stroke (yes vs. no), history of heart attack (yes vs. no), vegetables intake (continuous), fruit intake (continuous), alcohol drinking status (never vs. former vs. current), and total energy intake (continuous).

Subgroup analyses were performed based on sex, smoking status, and BMI. Sensitivity analyses were conducted by excluding events that occurred within 2 years or within 5 years of follow-up. Interaction assessments were tested using likelihood-ratio tests compared models with and without the interaction term. The proportional hazards $(\mathrm{PH})$ assumption was checked using the Schoenfeld residual test (18). Restricted cubic spline models (19) with three fitted knots (i.e., 10th, 50th, and 90th percentiles) were used to investigate the dose-response relationship between tomato or lycopene intake (as a continuous variable) and each outcome after full adjustment. All statistical analyses were performed using the software STATA version 15 (Stata Corp, College Station, TX, USA) with two-sided $P$-values. 
TABLE 3 | Associations between intake of raw tomato, tomato catsup or lycopene, and CVD mortality.

\begin{tabular}{|c|c|c|c|c|c|}
\hline Variables & Median & Cohort (n) & Cases (n) & HR $(95 \% \mathrm{Cl})^{\#}, p$-value & HR $(95 \% \mathrm{Cl})^{\star}, p$-value \\
\hline \multicolumn{6}{|l|}{ Raw tomato (g/day) } \\
\hline Q1 ( $\leq 3.63)$ & 1.63 & 20,508 & 1,645 & Reference group & Reference group \\
\hline Q2 ( $\geq 3.65-\leq 9.53)$ & 6.33 & 20,276 & 1,526 & $0.92(0.86-0.99), p=0.018$ & $0.96(0.90-1.04), p=0.327$ \\
\hline Q3 ( $\geq 9.55-\leq 17.56)$ & 12.91 & 20,619 & 1,491 & $0.89(0.83-0.95), p=0.001$ & $0.96(0.90-1.04), p=0.323$ \\
\hline Q4 ( $\geq 17.67-\leq 32.44)$ & 23.79 & 20,928 & 1,413 & $0.84(0.78-0.90), p<0.001$ & $0.90(0.83-0.97), p=0.006$ \\
\hline \multirow[t]{2}{*}{ Q5 ( $\geq 32.64)$} & 50.24 & 19,506 & 1,459 & $0.92(0.86-0.98), p=0.017$ & $0.95(0.88-1.03), p=0.248$ \\
\hline & & & & $p$ for trend $=0.063$ & $p$ for trend $=0.268$ \\
\hline \multicolumn{6}{|c|}{ Tomato catsup (g/day) } \\
\hline Q1 $(\leq 0.11)$ & 0 & 21,636 & 1,705 & Reference group & Reference group \\
\hline Q2 ( $\geq 0.13-\leq 0.44)$ & 0.17 & 19,575 & 1,328 & $0.93(0.86-1.00), p=0.045$ & $0.96(0.89-1.03), p=0.236$ \\
\hline Q3 $(\geq 0.48-\leq 1.15)$ & 0.58 & 21,243 & 1,400 & $0.89(0.83-0.95), p=0.001$ & $0.91(0.85-0.98), p=0.009$ \\
\hline Q4 ( $\geq 1.20-\leq 2.53)$ & 1.99 & 19,486 & 1,634 & $0.93(0.87-1.00), p=0.043$ & $0.96(0.89-1.03), p=0.285$ \\
\hline \multirow[t]{2}{*}{ Q5 ( $\geq 2.95)$} & 5.06 & 19,897 & 1,467 & $0.95(0.88-1.02), p=0.140$ & $0.92(0.85-0.99), p=0.029$ \\
\hline & & & & $p$ for trend $=0.903$ & $p$ for trend $=0.167$ \\
\hline \multicolumn{6}{|l|}{ Lycopene (mg/day) } \\
\hline Q1 (<2.79) & 2.07 & 20,368 & 1,688 & Reference group & Reference group \\
\hline Q2 ( $\geq 2.79-<4.06)$ & 3.42 & 20,367 & 1,494 & $0.91(0.85-0.98), p=0.011$ & $0.95(0.89-1.03), p=0.200$ \\
\hline Q3 $(\geq 4.06-<5.61)$ & 4.76 & 20,368 & 1,378 & $0.87(0.81-0.94), p<0.001$ & $0.91(0.85-0.98), p=0.017$ \\
\hline Q4 ( $\geq 5.61-<8.44)$ & 6.74 & 20,367 & 1,373 & $0.88(0.82-0.94), p<0.001$ & $0.90(0.83-0.98), p=0.012$ \\
\hline \multirow[t]{2}{*}{ Q5 ( $\geq 8.44)$} & 12.06 & 20,367 & 1,601 & $1.03(0.96-1.11), p=0.375$ & $1.01(0.93-1.11), p=0.742$ \\
\hline & & & & $p$ for trend $=0.033$ & $p$ for trend $=0.265$ \\
\hline
\end{tabular}

\#Adjusted for age (continuous) and sex (male vs. female).

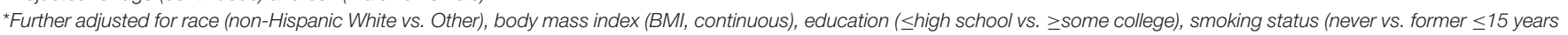

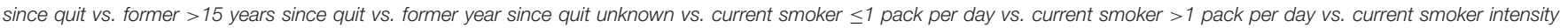

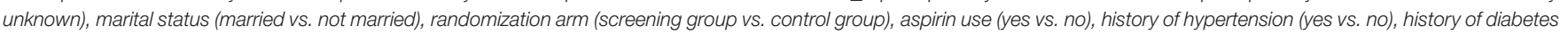

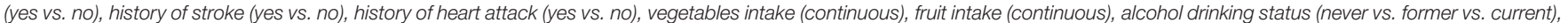
and total energy intake (continuous).

\section{RESULTS}

\section{Study Characteristic}

During a total of 1,672,715 follow-up years, 24,141 all-cause deaths, 7,534 CVD deaths and 7,161 cancer deaths occurred. The median (IQR) follow-up duration was 17.0 (15.0-19.0) years. The average age of participants at baseline was 62.4 (SD 5.3) years. The median intakes of raw tomato, tomato catsup, and lycopene were $12.91 \mathrm{~g} /$ day, $0.58 \mathrm{~g} / \mathrm{day}$, and $4.76 \mathrm{mg} /$ day, respectively. In comparison with participants in the lowest category of raw tomato, tomato catsup or lycopene, participants in the highest category were fatter, more likely to be non-Hispanic White and former smokers, and have a higher total energy intake (Table 1 and Supplementary Tables 1, 2).

\section{Tomato or Lycopene Intake and All-Cause Mortality}

Based on the most fully adjusted model 2, raw tomato intake was statistically significantly inversely associated with total mortality (Q5 vs. Q1; HR, 0.95 [95\% CI, 0.91-0.99]) (Table 2). Higher consumption of tomato catsup was significantly associated with a lower total mortality (Q5 vs. Q1; HR, 0.93 [95\% CI, 0.89-0.97]). Moderate dietary intake of lycopene was inversely linked with a reduced total mortality (Q4 vs. Q1; HR, 0.88 [95\% CI, 0.850.93])

\section{Tomato or Lycopene Intake and Cause-Specific Mortality}

Based on the most fully adjusted model 2, moderate dietary intake of raw tomato was statistically significantly associated with a lower CVD mortality (Q4 vs. Q1; HR, 0.90 [95\% CI, 0.830.97]) (Table 3). Increased consumption of tomato catsup was significantly associated with a reduced CVD mortality (Q5 vs. Q1; HR, 0.92 [95\% CI, 0.85-0.99]). Moderate dietary intake of lycopene was significantly related with a lower CVD mortality (Q4 vs. Q1; HR, 0.90 [95\% CI, 0.83-0.98]).

Based on the most fully adjusted model 2, dietary intake of raw tomato was not associated with cancer mortality (Q5 vs. Q1; HR, 1.04 [95\% CI, 0.96-1.14]) (Table 4). Higher consumption of tomato catsup was also not related with cancer mortality (Q5 vs. Q1; HR, 1.00 [95\% CI, 0.93-1.08]). Moderate dietary intake of lycopene was significantly associated with a lower cancer mortality (Q4 vs. Q1; HR, 0.89 [95\% CI, 0.82-0.96]).

\section{Restricted Cubic Spline Model Analysis}

There was a non-linear J-shaped association between raw tomato consumption and total mortality (magnitude of the relative reduction $=9 \%$; nadir at $31 \mathrm{~g} /$ day; $P$-value for non-linear association $<0.001$; Figure 1A). A J-shaped association was also observed for tomato catsup (magnitude of the relative reduction $=7 \%$; nadir at $3 \mathrm{~g} / \mathrm{d}$; $P$ for non-linearity $<0.001$; Figure $1 \mathrm{~B}$ ) and 
TABLE 4 | Associations between intake of raw tomato, tomato catsup or lycopene, and cancer mortality.

\begin{tabular}{|c|c|c|c|c|c|}
\hline Variables & Median & Cohort (n) & Cases (n) & HR $(95 \% \mathrm{Cl})^{\#}, p$-value & HR $(95 \% \text { Cl })^{\star}, p$-value \\
\hline \multicolumn{6}{|l|}{ Raw tomato (g/day) } \\
\hline Q1 ( $\leq 3.63)$ & 1.63 & 20,508 & 1,479 & Reference group & Reference group \\
\hline Q2 ( $\geq 3.65-\leq 9.53)$ & 6.33 & 20,276 & 1,439 & $0.97(0.90-1.05), p=0.452$ & $1.02(0.95-1.10), p=0.591$ \\
\hline Q3 ( $\geq 9.55-\leq 17.56)$ & 12.91 & 20,619 & 1,395 & $0.93(0.87-1.00), p=0.060$ & $1.01(0.93-1.09), p=0.869$ \\
\hline Q4 ( $\geq 17.67-\leq 32.44)$ & 23.79 & 20,928 & 1,449 & $0.96(0.89-1.03), p=0.289$ & $1.03(0.96-1.11), p=0.425$ \\
\hline \multirow[t]{2}{*}{ Q5 ( $\geq 32.64)$} & 50.24 & 19,506 & 1,399 & $0.98(0.91-1.06), p=0.615$ & $1.04(0.96-1.14), p=0.301$ \\
\hline & & & & $p$ for trend $=0.988$ & $p$ for trend $=0.301$ \\
\hline \multicolumn{6}{|c|}{ Tomato catsup (g/day) } \\
\hline Q1 $(\leq 0.11)$ & 0 & 21,636 & 1,512 & Reference group & Reference group \\
\hline Q2 ( $\geq 0.13-\leq 0.44)$ & 0.17 & 19,575 & 1,310 & $1.00(0.93-1.08), p=0.898$ & $1.02(0.94-1.10), p=0.656$ \\
\hline Q3 $(\geq 0.48-\leq 1.15)$ & 0.58 & 21,243 & 1,372 & $0.95(0.88-1.02), p=0.163$ & $0.97(0.90-1.05), p=0.478$ \\
\hline Q4 ( $\geq 1.20-\leq 2.53)$ & 1.99 & 19,486 & 1,494 & $0.96(0.89-1.04), p=0.308$ & $0.98(0.91-1.06), p=0.567$ \\
\hline \multirow[t]{2}{*}{ Q5 ( $\geq 2.95)$} & 5.06 & 19,897 & 1,473 & $1.02(0.95-1.10), p=0.568$ & $1.00(0.93-1.08), p=0.935$ \\
\hline & & & & $p$ for trend $=0.324$ & $p$ for trend $=0.907$ \\
\hline \multicolumn{6}{|l|}{ Lycopene (mg/day) } \\
\hline Q1 (<2.79) & 2.07 & 20,368 & 1,531 & Reference group & Reference group \\
\hline Q2 ( $\geq 2.79-<4.06)$ & 3.42 & 20,367 & 1,367 & $0.89(0.83-0.96), p=0.002$ & $0.91(0.85-0.98), p=0.016$ \\
\hline Q3 $(\geq 4.06-<5.61)$ & 4.76 & 20,368 & 1,411 & $0.93(0.86-1.00), p=0.037$ & $0.95(0.88-1.03), p=0.193$ \\
\hline Q4 ( $\geq 5.61-<8.44)$ & 6.74 & 20,367 & 1,346 & $0.88(0.82-0.95), p=0.001$ & $0.89(0.82-0.96), p=0.004$ \\
\hline \multirow[t]{2}{*}{ Q5 ( $\geq 8.44)$} & 12.06 & 20,367 & 1,506 & $0.98(0.91-1.06), p=0.617$ & $0.95(0.87-1.04), p=0.261$ \\
\hline & & & & $p$ for trend $=0.520$ & $p$ for trend $=0.677$ \\
\hline
\end{tabular}

\#Adjusted for age (continuous) and sex (male vs. female).

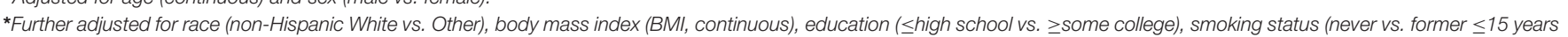

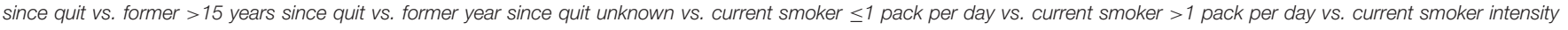

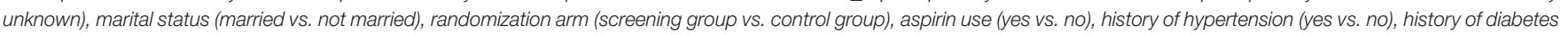

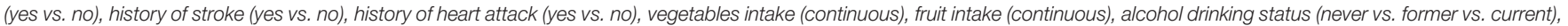
and total energy intake (continuous).

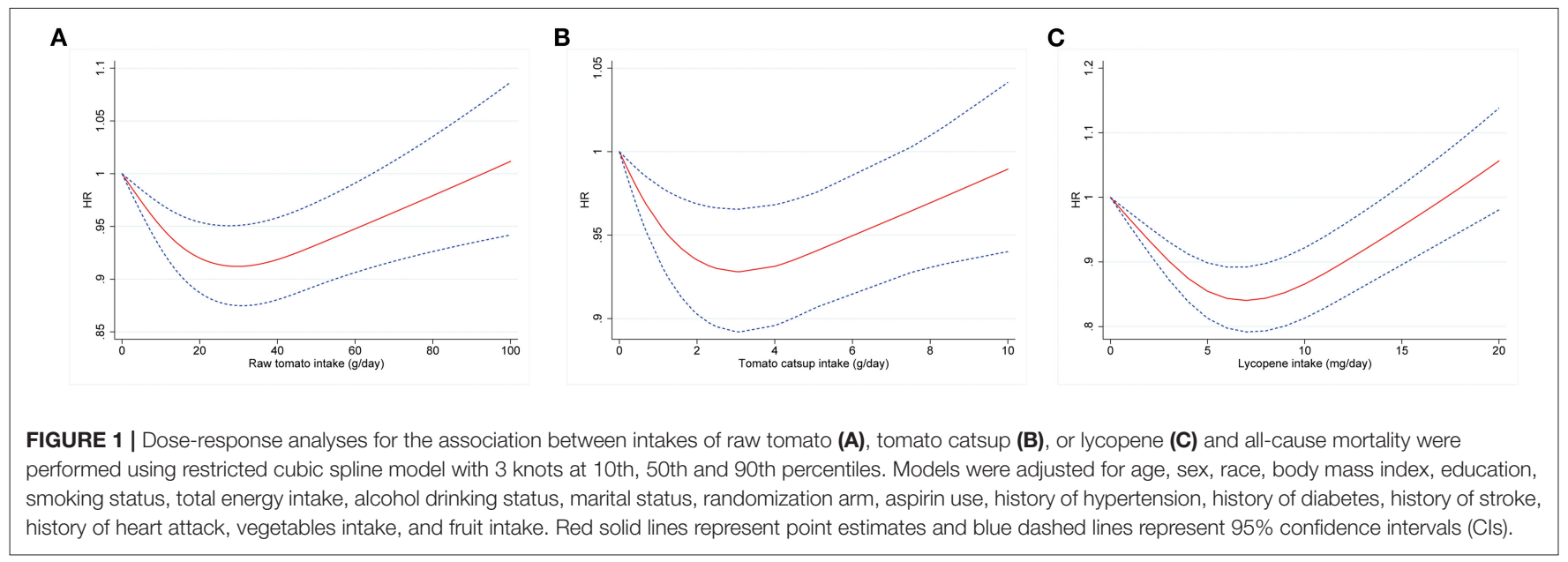

lycopene (magnitude of the relative reduction $=16 \%$; nadir at 7 $\mathrm{mg} /$ day; $P$ for non-linearity $<0.001$; Figure 1C).

\section{Additional Analyses}

The results of subgroup analyses are presented in Table 5. Tomato or lycopene intake remained consistently associated with reduced total mortality in almost all subgroups. An exception was represented by smoking status for tomato catsup. The association between tomato catsup intake and all-cause mortality risk was stronger in former cigarette smokers compared with that in current smokers $(P$ for interaction $=0.035$ ). In sensitivity analysis, results remained qualitatively similar after excluding events ascertained within 2 or 5 years (data not shown). Similar results were also obtained for total mortality when using ratio of tomato intake to total energy intake as exposure (raw tomato: Q5 vs. Q1; HR, 0.93 [95\% CI, 0.89-0.97]; tomato catsup: Q5 vs. Q1; 
TABLE 5 | Subgroup analyses of the associations between tomato or lycopene intake and total mortality were performed based on sex, smoking status, and BMI.

\begin{tabular}{|c|c|c|c|c|c|c|c|}
\hline Variables & Group & Raw tomato & $P$ for interaction & Tomato catsup & $P$ for interaction & Lycopene & $P$ for interaction \\
\hline \multirow[t]{5}{*}{ Male } & Q1 & Reference group & $>0.05$ & & $>0.05$ & & $>0.05$ \\
\hline & Q2 & $0.95(0.91-1.00), p=0.056$ & & $0.97(0.91-1.03), p=0.296$ & & $0.95(0.90-1.01), p=0.097$ & \\
\hline & Q3 & $0.94(0.89-0.99), p=0.021$ & & $0.92(0.87-0.98), p=0.006$ & & $0.93(0.88-0.99), p=0.017$ & \\
\hline & Q4 & $0.90(0.86-0.96), p<0.001$ & & $0.94(0.90-0.99), p=0.023$ & & $0.91(0.86-0.97), p=0.002$ & \\
\hline & Q5 & $0.95(0.90-1.00), p=0.059$ & & $0.93(0.88-0.98), p=0.007$ & & $1.01(0.95-1.08), p=0.671$ & \\
\hline \multirow[t]{5}{*}{ Female } & Q1 & Reference group & & & & & \\
\hline & Q2 & $0.97(0.90-1.03), p=0.308$ & & $0.92(0.87-0.98), p=0.004$ & & $0.88(0.83-0.94), p<0.001$ & \\
\hline & Q3 & $0.91(0.85-0.97), p=0.004$ & & $0.89(0.84-0.94), p<0.001$ & & $0.87(0.81-0.93), p<0.001$ & \\
\hline & Q4 & $0.92(0.86-0.99), p=0.017$ & & $0.93(0.85-1.00), p=0.064$ & & $0.85(0.79-0.91), p<0.001$ & \\
\hline & Q5 & $0.96(0.89-1.03), p=0.235$ & & $0.94(0.88-1.01), p=0.110$ & & $0.95(0.87-1.03), p=0.192$ & \\
\hline \multirow[t]{5}{*}{ Never smokers } & Q1 & Reference group & $>0.05$ & & 0.035 & & $>0.05$ \\
\hline & Q2 & $0.93(0.87-0.99), p=0.027$ & & $0.93(0.87-0.99), p=0.031$ & & $0.88(0.83-0.94), p<0.001$ & \\
\hline & Q3 & $0.89(0.83-0.95), p<0.001$ & & $0.91(0.85-0.97), p=0.003$ & & $0.87(0.81-0.93), p<0.001$ & \\
\hline & Q4 & $0.88(0.82-0.94), p<0.001$ & & $0.93(0.87-1.00), p=0.051$ & & $0.85(0.79-0.92), p<0.001$ & \\
\hline & Q5 & $0.93(0.87-1.01), p=0.075$ & & $0.95(0.89-1.02), p=0.132$ & & $0.94(0.87-1.02), p=0.148$ & \\
\hline \multirow[t]{5}{*}{ Current smokers } & Q1 & Reference group & & & & & \\
\hline & Q2 & $1.05(0.96-1.16), p=0.296$ & & $1.05(0.94-1.16), p=0.396$ & & $0.93(0.84-1.03), p=0.168$ & \\
\hline & Q3 & $0.96(0.86-1.07), p=0.441$ & & $0.94(0.84-1.04), p=0.223$ & & $0.92(0.82-1.03), p=0.133$ & \\
\hline & Q4 & $1.03(0.92-1.14), p=0.609$ & & $0.89(0.80-0.99), p=0.035$ & & $0.89(0.79-1.00), p=0.046$ & \\
\hline & Q5 & $0.97(0.87-1.09), p=0.668$ & & $0.98(0.88-1.09), p=0.713$ & & $0.95(0.84-1.08), p=0.463$ & \\
\hline \multirow[t]{5}{*}{ Former smokers } & Q1 & Reference group & & & & & \\
\hline & Q2 & $0.95(0.89-1.01), p=0.074$ & & $0.92(0.87-0.98), p=0.006$ & & $0.95(0.89-1.01), p=0.083$ & \\
\hline & Q3 & $0.94(0.89-1.00), p=0.044$ & & $0.89(0.84-0.95), p<0.001$ & & $0.93(0.87-0.98), p=0.014$ & \\
\hline & Q4 & $0.90(0.84-0.95), p<0.001$ & & $0.93(0.88-0.98), p=0.013$ & & $0.91(0.85-0.97), p=0.005$ & \\
\hline & Q5 & $0.95(0.89-1.01), p=0.125$ & & $0.89(0.84-0.95), p<0.001$ & & $1.04(0.97-1.12), p=0.288$ & \\
\hline \multirow[t]{5}{*}{$\mathrm{BMl}<25.0 \mathrm{~kg} / \mathrm{m}^{2}$} & Q1 & Reference group & $>0.05$ & & $>0.05$ & & $>0.05$ \\
\hline & Q2 & $0.94(0.88-1.01), p=0.076$ & & $0.90(0.84-0.96), p=0.002$ & & $0.87(0.82-0.93), p<0.001$ & \\
\hline & Q3 & $0.88(0.82-0.94), p<0.001$ & & $0.89(0.84-0.96), p=0.001$ & & $0.88(0.82-0.94), p<0.001$ & \\
\hline & Q4 & $0.92(0.85-0.98), p=0.017$ & & $0.90(0.84-0.97), p=0.004$ & & $0.84(0.77-0.90), p<0.001$ & \\
\hline & Q5 & $0.90(0.83-0.97), p=0.007$ & & $0.94(0.87-1.01), p=0.109$ & & $0.96(0.88-1.05), p=0.343$ & \\
\hline \multirow[t]{5}{*}{$\mathrm{BMI} \geq 25.0 \mathrm{~kg} / \mathrm{m}^{2}$} & Q1 & Reference group & & & & & \\
\hline & Q2 & $0.97(0.92-1.02), p=0.220$ & & $0.97(0.92-1.02), p=0.265$ & & $0.95(0.91-1.00), p=0.069$ & \\
\hline & Q3 & $0.95(0.91-1.00), p=0.053$ & & $0.92(0.88-0.97), p=0.002$ & & $0.93(0.88-0.98), p=0.008$ & \\
\hline & Q4 & $0.91(0.87-0.96), p<0.001$ & & $0.96(0.91-1.01), p=0.087$ & & $0.92(0.87-0.97), p=0.004$ & \\
\hline & Q5 & $0.98(0.93-1.04), p=0.490$ & & $0.95(0.90-1.00), p=0.037$ & & $1.03(0.97-1.09), p=0.336$ & \\
\hline
\end{tabular}

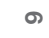

BMI, body mass index. 
HR, 0.94 [95\% CI, 0.90-0.97]; lycopene: Q4 vs. Q1; HR, 0.88 [95\% CI, 0.84-0.92]).

\section{DISCUSSION}

In this large, adult, US population, moderate intakes of raw tomato, tomato catsup, and lycopene were associated with reduced risks of all-cause and CVD mortality. Moderate lycopene intake was also inversely associated with cancer mortality. The associations of tomato and lycopene intake with all-cause mortality followed a non-linear J-shaped curve.

The observed protective associations of moderate tomato and lycopene consumption with total and CVD deaths were in line with a previous prospective study using data from the US National Health and Nutrition Examination Survey (NHANES) showing evidence of an inverse relationship between tomato or lycopene consumption and all-cause and CVD mortality (14). Our findings of null association between tomato intake and cancer mortality were not in accordance with Mazidi's study (20), which found that both tomato and lycopene intake were inversely related to cancer mortality based on NHANES data. By contrast, we only observed that moderate lycopene intake was significantly associated with cancer mortality.

In our study, we examined the impact of specific types of tomatoes (i.e., raw tomato and tomato catsup) on mortality. This was in contrast to a previous prospective study on this topic, which only examined associations with total tomato intake (14). Different types of tomatoes may have differential effects on health outcomes. Fraser et al. (21) reported that there was no relationship between intake of raw tomatoes and prostate cancer risk. However, a statistically significant multivariate-adjusted relationship between the intake of canned and cooked tomatoes and prostate cancer risk was observed. One bladder cancer study also found an inverse relationship with cooked, but not raw, tomatoes (22). Potential mechanisms by which cooking affects the association between tomato intake and cancer risk include changes in availability of some nutrients, destruction of digestive enzymes, and alteration of the structure and digestibility of food (23). In our study, both raw tomatoes and tomato catsup were statistically significantly inversely associated with total and CVD mortality.

Emerging evidence has suggested that tomato or lycopene intake may exert beneficial effects on human health, including some cancers (prostate, liver and stomach) $(12,13,24)$, metabolic syndrome (8), and CVD incidence (5). To the best of our knowledge, this is the first cohort study that has reported a J-shaped relationship between tomato or lycopene intake and health outcome. The possible underling mechanism is not clear and warrants further investigation. The findings of our study suggested that moderate consumption of tomato or lycopene was enough to reduce the all-cause and CVD deaths.

When we stratified results by smoking status, the association between tomato catsup intake and all-cause mortality risk was stronger in former smokers compared with that in current smokers $(P$ for interaction $=0.035)$, implying the potential residual confounding of smoking behavior. Thus, in multivariate analysis, we categorified the smoking status in more details.

Several potential mechanisms could explain the beneficial effects of moderate tomato or lycopene consumption on health, including strong antioxidant capacity to protect against oxidative stress (25), cholesterol reduction, modulation of inflammatory markers, metabolism to retinoids, and antiangiogenic effects (26). Interestingly, treatment with lycopene could reduce the formation of advanced glycation end products in HK-2 cells and in rat kidneys (27). Enhanced generation and accumulation of advanced glycation end products have been associated with an increased risk for CVD complications (28). Finally, various intervention trials have found beneficial effects on CVD risk markers, although the conflicts still exist (29).

The major strengths of this study included a large sample size of participants; a prospective cohort design; detailed information on diet and potential risk factors for deaths; and analyses on both tomato and lycopene intake. However, several limitations should be acknowledged. First, given the observational nature of our investigation, causality can only be suggested and residual confounding cannot be fully ruled out. Second, the vast majority of participants analyzed in this study were non-Hispanic Whites, which may limit its generalizability to other populations. Third, the J-shaped association may indicate the potential residual confounding of variables not included in the model (e.g., socialeconomic status). It is also possible that moderate tomato intake represents certain types of dietary pattern or intake of a good variety of vegetables. Lastly, participants' information was collected at baseline only and the exposures could have changed during the follow-up period.

In conclusion, in this large nationally representative sample of US adult population, intakes of raw tomato, tomato catsup and lycopene were associated with lower risks of all-cause and CVD mortality. Moderate consumption of lycopene was also related with a reduced cancer mortality. Further clinical studies and dietary intervention studies are warranted to confirm our premilitary findings.

\section{DATA AVAILABILITY STATEMENT}

The datasets presented in this study can be found in online repositories. The names of the repository/repositories and accession number(s) can be found at: https://cdas.cancer.gov/ datasets/plco/.

\section{ETHICS STATEMENT}

The studies involving human participants were reviewed and approved by National Cancer Institute. The patients/participants provided their written informed consent to participate in this study.

\section{AUTHOR CONTRIBUTIONS}

$\mathrm{XX}$ contributed to the conception or design of the work. XX, YZ, and SL contributed to the acquisition, 
analysis, or interpretation of data for the work. XX and SL drafted the manuscript. YZ critically revised the manuscript. All authors gave final approval and agree to be accountable for all aspects of work ensuring integrity and accuracy.

\section{FUNDING}

This study was supported by grants from the National Natural Science Foundation of China (81800588 and 81802520 ).

\section{REFERENCES}

1. Saini RK, Rengasamy KRR, Mahomoodally FM, Keum YS. Protective effects of lycopene in cancer, cardiovascular, and neurodegenerative diseases: an update on epidemiological and mechanistic perspectives. Pharmacol Res. (2020) 155:104730. doi: 10.1016/j.phrs.2020.104730

2. Petyaev IM, Dovgalevsky PY, Klochkov VA, Chalyk NE, Pristensky DV, Chernyshova MP, et al. Effect of lycopene supplementation on cardiovascular parameters and markers of inflammation and oxidation in patients with coronary vascular disease. Food Sci Nutr. (2018) 6:1770-7. doi: $10.1002 / \mathrm{fsn} 3.734$

3. Cheng HM, Koutsidis G, Lodge JK, Ashor A, Siervo M, Lara J. Tomato and lycopene supplementation and cardiovascular risk factors: a systematic review and meta-analysis. Atherosclerosis. (2017) 257:100-8. doi: 10.1016/j.atherosclerosis.2017.01.009

4. Li H, Chen A, Zhao L, Bhagavathula AS, Amirthalingam P, Rahmani J, et al. Effect of tomato consumption on fasting blood glucose and lipid profiles: a systematic review and meta-analysis of randomized controlled trials. Phytother Res. (2020) 34:1956-65. doi: 10.1002/pt r. 6660

5. Jacques PF, Lyass A, Massaro JM, Vasan RS, D'Agostino RB Sr. Relationship of lycopene intake consumption of tomato products to incident CVD. Br J Nutr. (2013) 110:545-51. doi: 10.1017/s00071145120 05417

6. Sesso HD, Liu S, Gaziano JM, Buring JE. Dietary lycopene, tomato-based food products and cardiovascular disease in women. J Nutr. (2003) 133:2336-41. doi: $10.1093 /$ jn/133.7.2336

7. Li N, Wu X, Zhuang W, Xia L, Chen Y, Wu C, et al. Tomato and lycopene and multiple health outcomes: umbrella review. Food Chem. (2021) 343:128396. doi: 10.1016/j.foodchem.2020.1 28396

8. Senkus KE, Tan L, Crowe-White KM. Lycopene and metabolic syndrome: a systematic review of the literature. Adv Nutr. (2019) 10:19-29. doi: 10.1093/advances/nmy069

9. Ratto F, Franchini F, Musicco M, Caruso G, Di Santo SG. A narrative review on the potential of tomato and lycopene for the prevention of Alzheimer's disease and other dementias. Crit Rev Food Sci Nutr. (2021) 1-12. doi: 10.1080/10408398.2021.1880363. [Epub ahead of print].

10. Rowles JL III, Ranard KM, Applegate CC, Jeon S, An R, Erdman JW Jr. Processed and raw tomato consumption and risk of prostate cancer: a systematic review and dose-response meta-analysis. Prostate Cancer Prostatic Dis. (2018) 21:319-36. doi: 10.1038/s41391-017-0005-x

11. Wang X, Yang HH, Liu Y, Zhou Q, Chen ZH. Lycopene consumption and risk of colorectal cancer: a meta-analysis of observational studies. Nutr Cancer. (2016) 68:1083-96. doi: 10.1080/01635581.2016.1206579

12. Yang T, Yang X, Wang X, Wang Y, Song Z. The role of tomato products and lycopene in the prevention of gastric cancer: a meta-analysis of epidemiologic studies. Med Hypotheses. (2013) 80:383-8. doi: 10.1016/j.mehy.2013.01.005

13. Thomas CE, Luu HN, Wang R, Adams-Haduch J, Jin A, Koh WP, et al. Association between dietary tomato intake and the risk of hepatocellular
ACKNOWLEDGMENTS

The authors thank the National Cancer Institute for access to NCI's data collected by the Prostate, Lung, Colorectal and Ovarian (PLCO) Cancer Screening Trial.

\section{SUPPLEMENTARY MATERIAL}

The Supplementary Material for this article can be found online at: https://www.frontiersin.org/articles/10.3389/fnut.2021. 684859/full\#supplementary-material carcinoma: the singapore chinese health study. Cancer Epidemiol Biomarkers Prev. (2020) 29:1430-5. doi: 10.1158/1055-9965.epi-20-0051

14. Mazidi M, Katsiki N, George ES, Banach M. Tomato and lycopene consumption is inversely associated with total and cause-specific mortality: a population-based cohort study, on behalf of the International Lipid Expert Panel (ILEP). Br J Nutr. (2020) 124:1303-10. doi: 10.1017/s0007114519002150

15. Prorok PC, Andriole GL, Bresalier RS, Buys SS, Chia D, Crawford ED, et al. Design of the Prostate, Lung, Colorectal and Ovarian (PLCO) cancer screening trial. Control Clin Trials. (2000) 21(Suppl. 6):273-309S doi: 10.1016/s0197-2456(00)00098-2

16. Subar AF, Thompson FE, Kipnis V, Midthune D, Hurwitz P, McNutt S, et al. Comparative validation of the Block, Willett, and National Cancer Institute food frequency questionnaires : the Eating at America's Table Study. Am J Epidemiol. (2001) 154:1089-99. doi: 10.1093/aje/154.12.1089

17. Subar AF, Midthune D, Kulldorff M, Brown CC, Thompson FE, Kipnis V, et al. Evaluation of alternative approaches to assign nutrient values to food groups in food frequency questionnaires. Am J Epidemiol. (2000) 152:279-86. doi: 10.1093/aje/152.3.279

18. Schoenfeld D. Chi-squared goodness-of-fit tests for the proportional hazards regression model. Biometrika. (1980) 67:145-53. doi: 10.1093/biomet/67.1.145

19. Marrie RA, Dawson NV, Garland A. Quantile regression and restricted cubic splines are useful for exploring relationships between continuous variables. J Clin Epidemiol. (2009) 62:511-7.e1. doi: 10.1016/j.jclinepi.2008. 05.015

20. Mazidi M, Ferns GA, Banach M. A high consumption of tomato and lycopene is associated with a lower risk of cancer mortality: results from a multi-ethnic cohort. Public Health Nutr. (2020) 23:1569-75. doi: $10.1017 / \mathrm{s} 1368980019003227$

21. Fraser GE, Jacobsen BK, Knutsen SF, Mashchak A, Lloren JI. Tomato consumption and intake of lycopene as predictors of the incidence of prostate cancer: the Adventist Health Study-2. Cancer Causes Control. (2020) 31:34151. doi: 10.1007/s10552-020-01279-z

22. Balbi JC, Larrinaga MT, De Stefani E, Mendilaharsu M, Ronco AL, Boffetta P, et al. Foods and risk of bladder cancer: a case-control study in Uruguay. Eur J Cancer Prev. (2001) 10:453-8. doi: 10.1097/00008469-20011000000009

23. Link LB, Potter JD. Raw versus cooked vegetables and cancer risk. Cancer Epidemiol Biomarkers Prev. (2004) 13:1422-35.

24. Xu X, Li J, Wang X, Wang S, Meng S, Zhu Y, et al. Tomato consumption and prostate cancer risk: a systematic review and meta-analysis. Sci Rep. (2016) 6:37091. doi: 10.1038/srep37091

25. Martí R, Roselló S, Cebolla-Cornejo J. Tomato as a source of carotenoids and polyphenols targeted to cancer prevention. Cancers. (2016) 8:58. doi: $10.3390 /$ cancers 8060058

26. Thies F, Mills LM, Moir S, Masson LF. Cardiovascular benefits of lycopene: fantasy or reality? Proc Nutr Soc. (2017) 76:122-9. doi: 10.1017/s0029665116000744

27. Tabrez S, Al-Shali KZ, Ahmad S. Lycopene powers the inhibition of glycationinduced diabetic nephropathy: a novel approach to halt the AGE-RAGE axis menace. Biofactors. (2015) 41:372-81. doi: 10.1002/biof.1238 
28. Stirban A, Gawlowski T, Roden M. Vascular effects of advanced glycation endproducts: clinical effects and molecular mechanisms. Mol Metab. (2014) 3:94-108. doi: 10.1016/j.molmet.2013. 11.006

29. Mordente A, Guantario B, Meucci E, Silvestrini A, Lombardi E, Martorana GE, et al. Lycopene and cardiovascular diseases: an update. Curr Med Chem. (2011) 18:1146-63. doi: 10.2174/092986711795029717

Disclaimer: The statements contained herein are solely those of the authors and do not represent or imply concurrence or endorsement by NCI.
Conflict of Interest: The authors declare that the research was conducted in the absence of any commercial or financial relationships that could be construed as a potential conflict of interest.

Copyright (C) $2021 \mathrm{Xu}, \mathrm{Li}$ and $\mathrm{Zhu}$. This is an open-access article distributed under the terms of the Creative Commons Attribution License (CC BY). The use, distribution or reproduction in other forums is permitted, provided the original author(s) and the copyright owner(s) are credited and that the original publication in this journal is cited, in accordance with accepted academic practice. No use, distribution or reproduction is permitted which does not comply with these terms. 